This is the post peer-review accepted manuscript of:

G. Abbasnejad and M. Carricato, "Direct Geometrico-static Problem of Underconstrained Cable-Driven Parallel Robots With n Cables," in IEEE Transactions on Robotics, vol. 31, no. 2, pp. 468- 478, April 2015. doi: 10.1109/TRO.2015.2393173

The published version is available online at:

https://ieeexplore.ieee.org/stamp/stamp.jsp?tp=\&arnumber $=7042311$

(C) 2015 IEEE. Personal use of this material is permitted. Permission from IEEE must be obtained for all other uses, in any current or future media, including reprinting/republishing this material for advertising or promotional purposes, creating new collective works, for resale or redistribution to servers or lists, or reuse of any copyrighted component of this work in other works. 


\section{Direct Geometrico-Static Problem of Underconstrained Cable-Driven Parallel Robots with $n$ Cables}

\author{
Ghasem Abbasnejad and Marco Carricato
}

\begin{abstract}
This paper studies the direct geometrico-static problem of underconstrained cable-driven parallel robots (CDPRs) supported by $n$ cables, with $n \leq 6$. The task consists in identifying the equilibrium poses of the end-effector when cable lengths are specified. The problem is challenging, because the end-effector preserves some freedoms after cable lengths are assigned by motors. Hence, kinematics and statics are coupled, and they must be tackled simultaneously. A general method is presented to model the problem by a set of algebraic equations, and a leastdegree univariate polynomial in the corresponding ideal is numerically found for any value of $n$. For the efficient computation of the solution set, a software is developed which implements an algorithm based on homotopy continuation. Distinctive features of the code are that it finds all problem solutions, including those with slack cables, and stability analysis is integrated in order to identify feasible configurations.
\end{abstract}

Index Terms-Cable-driven parallel robots, underconstrained robots, kinematic analysis, static analysis.

\section{INTRODUCTION}

In a cable-driven parallel robot (CDPR), a mobile platform is connected to the frame by $n$ cables, whose lengths are governed by motors. Since cables are active only when exert tensile axial forces, the number $m$ of cables that effectively contribute to controlling the platform pose may be instantaneously smaller than $n$. In a given configuration, a spatial CDPR is fully constrained if $m \geq 6$, since in this case the pose is completely determined by cable lengths and, thus, by motor inputs. On the contrary, a CDPR is underconstrained when $m<6$. In this occurrence, only $m$ degrees of freedom (dofs) of the platform are governed and the configuration of the robot depends on both the motor inputs and the external wrench acting on the platform. A CDPR is naturally underconstrained when $n<6$, whereas, when $n \geq 6$, it operates as such when mechanical equilibrium would require a negative tension in one or more cables. Though underconstrained CDPRs have received little attention in the literature $[1,2,3,4,5,6,7]$, a careful study of them is motivated. On the one hand, CDPRs with a limited number of cables may be used in several applications in which the task to be performed requires a limited number of controlled freedoms or a limitation of dexterity is acceptable in order to decrease complexity, cost, set-up time, likelihood of cable interference, etc. On the other hand, a CDPR with $n \geq 6$ may operate in underconstrained mode outside the wrenchclosure workspace $[8,9]$, thus increasing its operational capabilities if a suitable control is achieved.

Underconstrained CDPRs have distinguishing features. Due to the coupling between kinematics and statics (or dynamics), loop-closure and mechanical-equilibrium equations must be solved simultaneously and displacement-analysis problems become particularly challenging. Furthermore, since the platform is movable when cable lengths are assigned, stability plays a crucial role. An equilibrium configuration is actually feasible only if therein cable tensions are positive and equilibrium is stable. When multiple feasible equilibria exist, the platform may switch between them due to external disturbances, thus causing unpredicted motion.

G. Abbasnejad and M. Carricato are with the Dept. of Industrial Engineering, University of Bologna, Italy (e-mails: ghasem.abbasnejad2@unibo.it, marco.carricato@unibo.it).

This paper was presented in part at the 1st Int. Conf. on Cable-Driven Parallel Robots, Stuttgart, Germany, Sept. 2-4, 2012, and in part at the 6th Int. Workshop on Computational Kinematics, Barcelona, Spain, May 12-15, 2013.
Carricato and Merlet $[10,11]$ proposed a methodology for the kinematic, static and stability analysis of underconstrained CDPRs. By taking advantage of this approach, the direct (DGP) and the inverse geometrico-static problem (IGP) of CDPRs suspended by 2 and 3 cables were solved [11, 12, 13, 14, 15]. In a DGP, the static load and the cable lengths are specified, whereas the platform pose and the cable tensions must be calculated. In an IGP, the static load and $n$ desired platform coordinates are assigned, whereas the remaining ones, as well as the cable tensions and lengths, are to be computed.

This paper studies the DGP of underconstrained CDPRs suspended by 4,5 and 6 cables, with distinct anchor points on the base and the platform. The following simplifying assumptions are made: cables are inextensible and massless, interference is not taken into consideration, and the platform is acted upon by a constant force, e.g., gravity.

The problem is modeled by a set of algebraic equations, and a leastdegree univariate polynomial in the corresponding ideal is found. Though this polynomial proves to be too large to be numerically useful (it has degree 156, 216 and 140 for CDPRs being suspended by, respectively, 3, 4 and 5 cables), it has a twofold relevance. On the one hand, it sets an exact bound on the number of solutions of the DGP in the complex field (an information that is relevant for the algorithms that are implemented to compute the solution set). On the other hand, it provides a challenging benchmark to test the effectiveness of the elimination procedure presented in [12], thus showing its merits over other methods currently available to compute a least-degree univariate polynomial in a given ideal.

For the efficient computation of the solution set, a numerical procedure based on homotopy continuation is implemented in a software, DGP - Solver, which is freely distributed [16]. Distinctive features of the code are that it finds all solutions of the DGP, including those with slack cables, and stability analysis is integrated to identify feasible configurations. DGP - Solver uses Bertini [17] as a computation engine.

To show the potentiality of the software, an application of DGP - Solver to the optimal design of CDPRs is presented. Since a CDPR with $n \leq 6$ may admit multiple feasible equilibrium configurations, with some or all of them comprising slack cables, assessing the ability of a CDPR geometry to take advantage of all motorized cables to control the $n$ dofs of the platform is in order. An investigation providing preliminary ground in this respect is, thus, reported.

The structure of the contribution is as follows. Section II describes the geometrico-static model of the robot. Sections III and IV delineate the problem-solving elimination procedure and report the results obtained for generic CDPRs with $n \leq 6$. Section V presents the software DGP - Solver and the continuation procedure at its basis. Section VI gives a preliminary assessment of the ability of a CDPR to take full advantage of its motors. The paper provides a unifying framework for and expands the results presented at the conferences $[18,19]$. The software DGP - Solver is presented for the first time and Section VI is completely new.

In all reported numerical examples, measurements are expressed in SI units, with computations being performed on a PC with a $2.67 \mathrm{GHz}$ Intel Xeon processor and 4GB of RAM.

\section{GeOMETRICO-STATIC MODEL}

\section{A. Geometric and static model of the robot}

A mobile platform is connected to a fixed base by $n$ cables, with $n \leq 6$ (Fig. 1). The $i$ th cable, $i=1 \ldots n$, exits from the base at point $A_{i}$, and it is connected to the mobile platform at point $B_{i}$. The cable length is $\rho_{i}$, with $\rho_{i}>0$. $\mathcal{A}$ is a Cartesian frame attached to the base in $O$, with $\mathbf{i}, \mathbf{j}$ and $\mathbf{k}$ being unit vectors along the coordinate axes. $\mathcal{B}$ is a Cartesian frame attached to the platform in $G$, with $\mathbf{u}, \mathbf{v}$ and $\mathbf{w}$ 


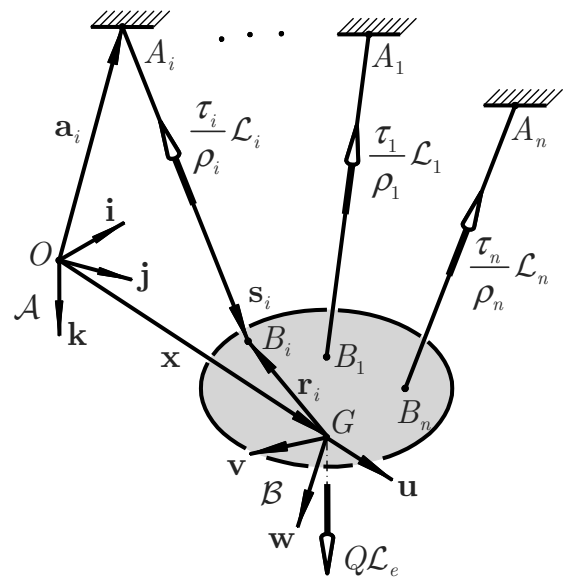

Fig. 1. A CDPR with $n$ cables: geometric and static model.

being unit vectors along its axes. $\mathbf{x}=[x, y, z]^{T}$ is the position vector of $G$ in $\mathcal{A}$, whereas $\mathbf{R}$ is the rotation matrix representing the orientation of $\mathcal{B}$ in $\mathcal{A}$. Furthermore, $\mathbf{a}_{i}=A_{i}-O$, and $\mathbf{s}_{i}=B_{i}-A_{i}=\mathbf{x}+\mathbf{R} \mathbf{b}_{i}-\mathbf{a}_{i}$, with $\mathbf{b}_{i}$ being the position vector of $B_{i}$ in $\mathcal{B}$.

The platform is acted upon by a constant force, which is assumed to be oriented as $\mathbf{k}$ and applied at $G$, without loss of generality. This force is described by a 0-pitch wrench $Q \mathcal{L}_{e}$, where $Q$ is the intensity of the force and $\mathcal{L}_{e}$ is the normalized Plücker vector of the force line of action. Typically, $Q \mathcal{L}_{e}$ is the gravity wrench, applied to the center of mass $G$. The normalized Plücker vector of the line associated with the $i$ th cable is $\mathcal{L}_{i} / \rho_{i}$, where, in axis coordinates, $\mathcal{L}_{i}=$ $-\left[\mathbf{s}_{i} ;\left(B_{i}-P\right) \times \mathbf{s}_{i}\right]$ and $B_{i}-P$ is a vector directed from a reference point $P$, called for brevity moment pole, to the cable line. $P$ may be chosen arbitrarily, and need not coincide with $O$ or $G$. The wrench exerted by the $i$ th cable on the platform is $\left(\tau_{i} / \rho_{i}\right) \mathcal{L}_{i}$, with $\tau_{i}$ being a positive scalar representing the intensity of the cable tensile force.

If all cables are in tension, the geometrical constraints on the platform are $\left\|\mathbf{s}_{i}\right\|^{2}=\rho_{i}^{2}, i=1 \ldots n$, and thus, by subtracting the first relation from the others,

$$
\begin{aligned}
& q_{1}:=\left\|\mathbf{s}_{1}\right\|^{2}-\rho_{1}^{2}=0 \\
& q_{j}:=\left\|\mathbf{s}_{j}\right\|^{2}-\left\|\mathbf{s}_{1}\right\|^{2}-\rho_{j}^{2}+\rho_{1}^{2}=0, \quad j=2 \ldots n
\end{aligned}
$$

where symbols $q_{1}, \ldots, q_{n}$ denote the polynomials at the left-hand side of the relations in Eq. (1).

Since only $n$ geometrical restraints are enforced, the platform preserves $6-n$ dofs, with its final pose being determined by static equilibrium. This may be written as $[10,11]$

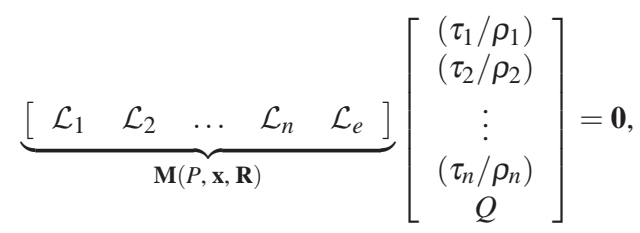

where $\tau_{i} \geq 0, i=1 \ldots n$, and $\mathbf{M}(P, \mathbf{x}, \mathbf{R})$ is a $6 \times(n+1)$ matrix depending on both the moment pole $P$ and the platform pose (here described by vector $\mathbf{x}$ and matrix $\mathbf{R}$ ), namely

$$
\mathbf{M}(P, \mathbf{x}, \mathbf{R})=\left[\begin{array}{cccc}
-\mathbf{s}_{1} & \cdots & -\mathbf{s}_{n} & \mathbf{k} \\
\left(P-B_{1}\right) \times \mathbf{s}_{1} & \cdots & \left(P-B_{n}\right) \times \mathbf{s}_{n} & (G-P) \times \mathbf{k}
\end{array}\right]
$$

Equations (1) and (2) form a system in the platform coordinates and the cable tensions. Three distinct pose parametrization are introduced in the following, with all of them leading to an algebraic formulation of the problem. Each parametrization has its own merits, and may be preferred to others depending on the problem-solving algorithm that is implemented.

\section{B. 6-parameter representation of the platform pose}

An algebraic representation $\mathbf{X}$ of the pose with a minimal set of parameters may be obtained by employing position vector $\mathbf{x}$ and the array $\Phi=\left[e_{1}, e_{2}, e_{3}\right]^{T}$ grouping the Rodrigues parameters of the platform orientation, i.e. $\mathbf{X}=\left[x, y, z, e_{1}, e_{2}, e_{3}\right]^{T}$. Rodrigues parameters emerge from Euler homogeneous variables $e_{0}, e_{1}, e_{2}$ and $e_{3}$ by the normalization $e_{0}=1$ [20]. When Euler/Rodrigues parameters are used, the rotation matrix $\mathbf{R}$ has the form

$$
\mathbf{R}=\mathbf{I}_{3}+2\left(e_{0} \tilde{\Phi}+\tilde{\Phi} \tilde{\Phi}\right) /\left(e_{0}^{2}+e_{1}^{2}+e_{2}^{2}+e_{3}^{2}\right)
$$

where $\tilde{\Phi}$ is the skew-symmetric matrix expressing the operator $\Phi \times$. In this case, Eqs. (1) and (2) form a system of $6+n$ polynomial equations in $6+n$ unknowns, i.e. $x, y, z, e_{1}, e_{2}, e_{3}, \tau_{1}, \ldots, \tau_{n}$. This formulation is particularly suitable when the DGP is solved by elimination techniques (cf. Section III), since it introduces the smallest number of unknowns.

\section{Study representation of the platform pose}

Study soma coordinates [20] provide a 8-parameter homogeneous representation of the pose, i.e. $\mathbf{S}=\left[e_{0}, e_{1}, e_{2}, e_{3}, g_{0}, g_{1}, g_{2}, g_{3}\right]^{T}$, where $e_{k}, k=0 \ldots 3$, are the Euler parameters of the platform orientation, normalized with

$$
e_{0}^{2}+e_{1}^{2}+e_{2}^{2}+e_{3}^{2}=1
$$

and $g_{k}, k=0 \ldots 3$, are the components of a quaternion such that

$$
e_{0} g_{0}+e_{1} g_{1}+e_{2} g_{2}+e_{3} g_{3}=0
$$

$\mathbf{R}$ is given by Eq. (4), whereas the platform position is

$$
\mathbf{x}=\frac{1}{e_{0}^{2}+e_{1}^{2}+e_{2}^{2}+e_{3}^{2}}\left[\begin{array}{l}
-e_{0} g_{1}+e_{1} g_{0}-e_{2} g_{3}+e_{3} g_{2} \\
-e_{0} g_{2}+e_{1} g_{3}+e_{2} g_{0}-e_{3} g_{1} \\
-e_{0} g_{3}-e_{1} g_{2}+e_{2} g_{1}+e_{3} g_{0}
\end{array}\right]
$$

Study coordinates add 2 unknowns and 2 equations to Eqs. (1) and (2), thus yielding a system of $8+n$ polynomial equations in $8+n$ unknowns, i.e. $e_{0}, e_{1}, e_{2}, e_{3}, g_{0}, g_{1}, g_{2}, g_{3}, \tau_{1}, \ldots, \tau_{n}$. This formalization is used in Section V-A to solve the DGP by general homotopy continuation.

\section{Dietmaier representation of the platform pose}

Following [21], the platform pose may be described by 9 variables, i.e. $\mathbf{D}=\left[\mathbf{x}^{T}, \mathbf{u}^{T}, \mathbf{v}^{T}\right]^{T}=\left[x, y, z, u_{1}, u_{2}, u_{3}, v_{1}, v_{2}, v_{3}\right]^{T}$, where $\left[u_{1}, u_{2}\right.$, $\left.u_{3}\right]$ and $\left[v_{1}, v_{2}, v_{3}\right]$ are, respectively, the components of $\mathbf{u}$ and $\mathbf{v}$ in $\mathcal{A}$, satisfying the conditions

$$
u_{1}^{2}+u_{2}^{2}+u_{3}^{2}=1, \quad v_{1}^{2}+v_{2}^{2}+v_{3}^{2}=1, \quad u_{1} v_{1}+u_{2} v_{2}+u_{3} v_{3}=0
$$

By setting $\mathbf{w}=\mathbf{u} \times \mathbf{v}$, the rotation matrix is given by $\mathbf{R}=[\mathbf{u}, \mathbf{v}, \mathbf{w}]$.

Equations (1), (2) and (8) form a system of $9+n$ polynomial equations in $9+n$ unknowns, i.e. $x, y, z, u_{1}, u_{2}, u_{3}, v_{1}, v_{2}, v_{3}, \tau_{1}, \ldots, \tau_{n}$. This formalization is used in Section V-A to solve the DGP by parameter homotopy continuation. 


\section{THE ELIMINATION ALGORITHM}

When an elimination technique is adopted, all variables but one need to be eliminated from the equations governing the problem, until a univariate polynomial is obtained. If the latter has the least possible degree, it provides the exact number of solutions in the complex field. For elimination to be successful, a formulation containing the least number of unknowns is often the most suitable. Thus, the formulation introduced in Section II-B is used in this case.

Cable tensions may be eliminated as suggested in [10, 11]. In fact, Eq. (2) admits nonzero solutions only if

$$
\operatorname{rank}[\mathbf{M}(P, \mathbf{X})] \leq n
$$

i.e. if $\mathcal{L}_{1}, \ldots, \mathcal{L}_{n}$ and $\mathcal{L}_{e}$ are linearly dependent. This is a purely geometric condition, since $\mathbf{M}(P, \mathbf{X})$ only depends on the moment pole $P$ and the platform pose, now described by the array $\mathbf{X}=[\mathbf{x}, \Phi]$. By setting all $(n+1) \times(n+1)$ minors of $\mathbf{M}(P, \mathbf{X})$ to zero and by varying $P$ (the latter may be chosen arbitrarily), a large set of scalar relations that do not contain cable tensions may be obtained (cf. Sections IV-B and IV-C), namely

$$
p_{k}(\mathbf{X})=0, \quad k=1 \ldots h,
$$

where $p_{1}, \ldots, p_{h}$ denote polynomials in $\mathbf{X}$.

Since Eqs. (1) and (10) allow the platform pose to be completely determined, the DGP solution emerges as the affine variety of the ideal $\langle I\rangle$ generated by $I=\left\{q_{1}, \ldots, q_{n}, p_{1}, \ldots, p_{h}\right\} .{ }^{1}$ For each pose $\mathbf{X}$, cable tensions may be successively calculated from $n$ relations suitably chosen within Eq. (2). Clearly, only the configurations for which all tensions are nonnegative must be retained. Stability may be determined by the algorithm presented in [11], by assessing the definiteness of a reduced Hessian matrix, $\mathbf{H}_{r}$.

Since the number $h$ of relations in Eq. (10) is ordinarily greater than $6-n$, relations $p_{k}$ may not be independent, otherwise the problem would admit no solution. However, most of them are dependent in a nonlinear way. The abundance of linearly-independent equations obtained by this strategy is particularly beneficial when the problem is solved by elimination procedures based on Groebner bases [22] and/or Sylvester dialytic approach [23].

The elimination algorithm presented in this section was first used in [12] to solve the DGP of a CDPR suspended by $n=3$ cables. Here, it is proved to be effectual for the cases $n=4$ and $n=5$, too. The procedure encompasses three steps.

1) Computation of an initial Groebner basis: For a generic CDPR with $n$ cables, $n \leq 6$, the number of monomials in $\mathbf{X}$ comprised in $I$ is of the order of several hundreds. Hence, computing a Groebner basis is a nontrivial task. The following expedients are adopted.

- To allow computation via the GroebnerPackage provided within Maple15, all geometric parameters of the robot are assigned generic rational values, i.e. $\langle I\rangle \subset \mathbb{Q}[\mathbf{X}]$, where $\mathbb{Q}[\mathbf{X}]$ is the set of all polynomials in $\mathbf{X}$ with coefficients in $\mathbb{Q}^{2}$ ' 'Generic' values are numbers such that the stated properties of $\langle I\rangle$ hold 'with algebraic probability one' in the sense specified in [25].

\footnotetext{
${ }^{1}$ Given the set of polynomials $I=\left\{f_{1}(\mathbf{X}), \ldots, f_{s}(\mathbf{X})\right\}$, the ideal $\langle I\rangle$ is the set of all polynomials obtained as $\sum_{i=1 \ldots .} h_{i}(\mathbf{X}) f_{i}(\mathbf{X})$, where $h_{i}(\mathbf{X})$ is an arbitrary polynomial in the same field as $f_{1}, \ldots, f_{s}$. Loosely speaking, $\langle I\rangle$ is the span obtained by linearly combining $f_{1}, \ldots, f_{s}$ by using polynomial coefficients. $f_{1}, \ldots, f_{s}$ form a so-called basis of $\langle I\rangle$. Recalling that the variety $V(I)$ of $I$ is the set of all $\mathbf{X}$ such that $f_{1}(\mathbf{X})=\ldots=f_{s}(\mathbf{X})=0, V$ depends only on the ideal generated by $I$, i.e. $V(I)=V(\langle I\rangle)[22]$. Thus, changing the basis of $\langle I\rangle$ may allow an easier find of the zeros of the polynomials in $I$.

${ }^{2}$ The computation of a Groebner basis cannot be executed in floating-point arithmetic by the standard approaches currently implemented in computer algebra systems, due to the basis instability under small changes in the coefficients of the system (see, for example, [24]).
}

- Groebner bases are computed with respect to graded reverse lexicographic monomial orders (grevlex, in brief), which provide the most efficient calculations.

- The abundance of generators in $I$ is fully exploited, since it speeds up calculation (a feature already pointed out in [26]).

By the above expedients, a Groebner basis $G[I]$ of $\langle I\rangle$ with respect to grevlex $(\mathbf{X})$, with variables ordered so that $z>y>x>e_{1}>e_{2}>e_{3}$, may be generally computed in a fairly expedited way. Once $G[I]$ is known, the normal set $\mathbf{N}[I]$ of $\langle I\rangle$, i.e. the set of all monomials that are not multiples of any leading monomial in $G[I]$, may be easily computed [27]. For the properties of Groebner bases, the number of monomials in $\mathbf{N}[I]$ coincides with the number $N_{\text {sol }}$ of solutions of the problem at hand and, thus, with the order of the least-degree univariate polynomials in $\langle I\rangle$.

2) Computation of elimination ideals: If $\mathbf{X}_{l}$ is a list of $l$ variables in $\mathbf{X}$ and $\mathbf{X} \backslash \mathbf{X}_{l}$ is the (ordered) relative complement of $\mathbf{X}_{l}$ in $\mathbf{X}$, the lth elimination ideal $\left\langle I_{l}\right\rangle$ of $\langle I\rangle$ is defined as $\langle I\rangle \cap \mathbb{Q}\left[\mathbf{X} \backslash \mathbf{X}_{l}\right]$, namely as the subset of all polynomials of $\langle I\rangle$ that comprise variables in $\mathbf{X} \backslash \mathbf{X}_{l}$ only (and in which, thus, the $l$ variables in $\mathbf{X}_{l}$ have been eliminated). As $\langle I\rangle$ comprises 6 unknowns, the polynomials of $\left\langle I_{1}\right\rangle$ contain 5 variables, the polynomials of $\left\langle I_{2}\right\rangle$ only 4 , and so on. $\left\langle I_{5}\right\rangle$ comprises a single variable and, thus, it contains the least-degree polynomial of $\langle I\rangle$ in that variable. In theory, once $G[I]$ is known, one may use the Faugère-Gianni-Lazard-Mora (FGLM) algorithm [28] to compute the Groebner basis $G\left[I_{l}\right]$ of $\left\langle I_{l}\right\rangle$ with respect to grevlex $\left(\mathbf{X} \backslash \mathbf{X}_{l}\right)$ for any $l$. In practice, however, computing $\left\langle I_{l}\right\rangle$ is very demanding in terms of both computation time and memory usage. Performing the elimination of the 'last' variables is extremely onerous and is likely to prove unfeasible on normal workstations $\left(G\left[I_{5}\right]\right.$ is the desired least-degree univariate polynomial). A far more efficient alternative consists in using the FGLM algorithm to eliminate only a subset of the original unknowns, thus computing $G\left[I_{l}\right]$ for some $l$, and then completing the elimination process by applying a dialytic step to the polynomials of $G\left[I_{l}\right]$, as discussed in the next step.

3) Final dialytic elimination: Dhingra et al. [26] proposed a dialytic method to compute $G\left[I_{5}\right]$ from $G[I]$ without deriving elimination ideals. In this case, Dhingra's method, however, is not efficient, since the eliminant matrix that is obtained from $G[I]$ is too large for its expansion to be performed in a reasonable time. A feasible alternative emerges by observing that, for the problem at hand, dialytic elimination may be applied to the Groebner basis of any elimination ideal of $\langle I\rangle$. In fact, if $e_{3}$ is the smallest variable in $\mathbf{X} \backslash \mathbf{X}_{l}, G\left[I_{l}\right]$ comprises a number of monomials in $\mathbf{X} \backslash \mathbf{X}_{l}-\left\{e_{3}\right\}$ that is ordinarily equal to the number $N_{l}$ of generators in $G\left[I_{l}\right]$, for all values of $l$. It follows that the generators of $G\left[I_{l}\right]$ may be set up, for any $l$, as a square system of homogeneous linear equations in the monomials of $\mathbf{X}-\left\{e_{3}\right\}$. A Sylvester-type eliminant matrix $\mathbf{E}_{l}\left(e_{3}\right)$, whose elements only depend on $e_{3}$, may thus be constructed, and a resultant in $e_{3}$, free from extraneous polynomial factors, computed, i.e. $G\left[I_{5}\right]=\operatorname{det} \mathbf{E}_{l}\left(e_{3}\right)$.

The above elimination procedure was applied to several robot geometries, with 3, 4 and 5 cables. Throughout the numerical experimentation, the fastest derivation of $G\left[I_{5}\right]$ was always obtained by computing $G\left[I_{3}\right]$ by the FGLM algorithm, thus eliminating $\{x, y, z\}$, and applying a final dialytic step to $G\left[I_{3}\right]$, thus eliminating $\left\{e_{1}, e_{2}\right\}$.

\section{DGP OF CDPRS WITH $n$ CABLES}

Hereafter, the application of the elimination procedure to the DGP of generic CDPRs suspended by 4 and 5 cables is discussed in detail. The DGP of CDPRs with 2, 3 and 6 cables is presented briefly, since its detailed description is available elsewhere. 


\section{A. DGP of CDPRs with 2 and 3 cables}

Carricato and Merlet [11] showed that the DGP of the CDPR with 2 cables may be formulated by way of two planer models. In each model, the DGP is solved by a 12-degree univariate polynomial, so that, on the whole, the DGP admits 24 solutions in the complex field, all of which may be real.

The DGP of the CDPR with 3 cables is described in detail in [12]. Equation (1) provides 3 polynomials, respectively of degree 4, 3 and 3 in $\mathbf{X}$, whereas Eq. (10) yields 11 polynomials of degree 6 in $\mathbf{X}$, so that $I=\left\{q_{1}, q_{2}, q_{3}, p_{1}, \ldots, p_{11}\right\}$. A Groebner basis $G[I]$ of $\langle I\rangle$ may be computed efficiently with respect to $\operatorname{grevlex}\left(z, y, x, e_{1}, e_{2}, e_{3}\right)$. A univariate polynomial of degree 156 in $e_{3}$ is computed by calculating $\left\langle I_{3}\right\rangle$ via the FGLM algorithm and by successively expanding a $45 \times 45$ eliminant matrix. Though the problem admits exactly 156 solutions in the complex field, sets of robot parameters for which the DGP provides no more than 54 real configurations were found so far [14].

\section{B. DGP of CDPRs with 4 cables}

When all 4 cables are taut, Eq. (1) yields 4 polynomials, i.e. $q_{1}$, $q_{2}, q_{3}$ and $q_{4}$, respectively of degree $4,3,3$ and 3 in $\mathbf{X}$. As far as the static constraints are concerned, when $P \equiv A_{1}$, all equations emerging by setting the $5 \times 5$ minors of $\mathbf{M}\left(A_{1}, \mathbf{X}\right)$ to zero, namely ${ }^{3}$

$$
\begin{array}{ll}
p_{1}:=\operatorname{det} \mathbf{M}_{23456}\left(A_{1}, \mathbf{X}\right)=0, & p_{2}:=\operatorname{det} \mathbf{M}_{13456}\left(A_{1}, \mathbf{X}\right)=0 \\
p_{3}:=\operatorname{det} \mathbf{M}_{12456}\left(A_{1}, \mathbf{X}\right)=0, & p_{4}:=\operatorname{det} \mathbf{M}_{12356}\left(A_{1}, \mathbf{X}\right)=0 \\
p_{5}:=\operatorname{det} \mathbf{M}_{12346}\left(A_{1}, \mathbf{X}\right)=0, & p_{6}:=\operatorname{det} \mathbf{M}_{12345}\left(A_{1}, \mathbf{X}\right)=0
\end{array}
$$

are linearly independent. The relations obtained by letting $P \equiv A_{i}, i=$ $2 \ldots 4$, are linearly dependent on those in Eq. (11), so that they may be discarded. Nine additional linearly-independent equations may be obtained by letting $P \equiv B_{i}, i=1 \ldots 4$, namely

$$
\begin{aligned}
& p_{7}:=\operatorname{det} \mathbf{M}_{23456}\left(B_{1}, \mathbf{X}\right)=0, p_{8}:=\operatorname{det} \mathbf{M}_{13456}\left(B_{1}, \mathbf{X}\right)=0 \\
& p_{9}:=\operatorname{det} \mathbf{M}_{12456}\left(B_{1}, \mathbf{X}\right)=0, p_{10}:=\operatorname{det} \mathbf{M}_{23456}\left(B_{2}, \mathbf{X}\right)=0 \\
& p_{11}:=\operatorname{det} \mathbf{M}_{13456}\left(B_{2}, \mathbf{X}\right)=0, p_{12}:=\operatorname{det} \mathbf{M}_{12456}\left(B_{2}, \mathbf{X}\right)=0(12) \\
& p_{13}:=\operatorname{det} \mathbf{M}_{23456}\left(B_{3}, \mathbf{X}\right)=0, p_{14}:=\operatorname{det} \mathbf{M}_{13456}\left(B_{4}, \mathbf{X}\right)=0 \\
& p_{15}:=\operatorname{det} \mathbf{M}_{12456}\left(B_{5}, \mathbf{X}\right)=0
\end{aligned}
$$

and two more by letting $P \equiv G$, i.e.

$$
p_{16}:=\operatorname{det} \mathbf{M}_{23456}(G, \mathbf{X})=0, \quad p_{17}:=\operatorname{det} \mathbf{M}_{13456}(G, \mathbf{X})=0
$$

All polynomials $p_{j}, j=1 \ldots 17$, have degree 6 in $\Phi$, degree 3 in $\mathbf{x}$ and total degree 9 in $\mathbf{X}$. No other linearly independent relations in $\mathbf{X}$ may be obtained from the minors of $\mathbf{M}(P, \mathbf{X})$ by varying the moment pole $P . I=\left\{q_{1}, q_{2}, q_{3}, q_{4}, p_{1}, \ldots, p_{17}\right\}$ comprises up to 1576 monomials in $\mathbf{X}$.

By taking advantage of the abundance of generators in $I$, the computation of $G[I]$ for the exemplifying robot whose dimensions are reported in subsequent Table II requires roughly $17 \mathrm{~min}$, which is a rather limited time for a problem of this size. Once $G[I]$ is known, the normal set of $\langle I\rangle$ may be computed, namely (in vector format)

$$
\mathbf{N}[I]=\left[1, e_{3}, e_{2}, e_{1}, x, y, z, e_{3}^{2}, e_{2} e_{3}, \ldots, e_{1} e_{2} e_{3}^{2} y\right]^{T} .
$$

Since $\mathbf{N}[I]$ comprises 216 monomials, $N_{\text {sol }}=216$.

The structure of $G[I]$ with respect to $\operatorname{grevlex}(\mathbf{X})$ is reported in Table I, as well as the structure of the Groebner bases $G\left[I_{l}\right]$ with respect to grevlex $\left(\mathbf{X} \backslash \mathbf{X}_{l}\right)$ of the elimination ideals $\left\langle I_{l}\right\rangle, l=1 \ldots 5$. These are derived from $G[I]$ by the FGLM algorithm. Column 3 reports the number $N_{l}$ of generators in $G\left[I_{l}\right]$, column 4 provides the degree in $\mathbf{X} \backslash \mathbf{X}_{l}$ of such generators (in parentheses, the number of

\footnotetext{
${ }^{3}$ The notation $\mathbf{M}_{h i j k l}(P, \mathbf{X})$ denotes the block matrix obtained from rows $h, i, j, k$ and $l$ of $\mathbf{M}(P, \mathbf{X})$.
}

generators having a given degree is specified), and column 5 reports, for each variable $w \in \mathbf{X} \backslash \mathbf{X}_{l}$, the number of monomials in $G\left[I_{l}\right]$ having variables in $\mathbf{X} \backslash \mathbf{X}_{l}-\{w\}$. Table I shows that, if $w$ is the smallest variable in $\mathbf{X} \backslash \mathbf{X}_{l}$, i.e. $e_{3}, G\left[I_{l}\right]$ comprises a number of monomials in $\mathbf{X} \backslash \mathbf{X}_{l}-\left\{e_{3}\right\}$ which is equal to $N_{l}$ for all values of $l$. For instance, the Groebner basis $G\left[I_{3}\right]$ of $\langle I\rangle \cap \mathbb{Q}\left[e_{1}, e_{2}, e_{3}\right]$ with respect to grevlex $\left(e_{1}\right.$, $\left.e_{2}, e_{3}\right)$ comprises 61 polynomials ( 1 of degree 8 in $\Phi$ and 60 of degree 10 in $\Phi$ ), including 61 monomials in $e_{1}$ and $e_{2}$.

Table II reports the CPU time required to compute $G\left[I_{l}\right]$ for an exemplifying generic robot. In particular, the third column reports the CPU time $T_{G\left[I_{l}\right]}$ required to obtain $G\left[I_{l}\right]$ from $\left\langle I_{l-1}\right\rangle$ by the FGLM algorithm. As expected, the higher $l$ (i.e. the more variables are eliminated), the longer the time necessary to perform the computation and, mainly, the larger the amount of memory that is required. In particular, the last elimination ideal cannot be computed, due to excessive memory usage.

The advantage gained by applying a dialytic step to a Groebner basis $G\left[I_{l}\right]$ with $l>0$ emerges from the data presented in the fourth column, which reports the CPU time $T_{G\left[I_{5}\right]}$ required to calculate $G\left[I_{5}\right]$ by applying Sylvester elimination to $G\left[I_{l}\right]$, for $l=0 \ldots 4$. Since the computation time of the dialytic step depends on the dimension of $\mathbf{E}_{l}$, it decreases with the number of variables in grevlex $\left(\mathbf{X} \backslash \mathbf{X}_{l}\right)$. For the example at hand, $G\left[I_{5}\right]$ cannot be computed from $G\left[I_{l}\right]$, with $l=0 \ldots 2$, due to excessive computation time. Instead, a univariate polynomial $G\left[I_{5}\right]$ in $e_{3}$ may be successfully computed from either $G\left[I_{3}\right]$ or $G\left[I_{4}\right]$. The most efficient computation is obtained by eliminating $\{x, y, z\}$ by the FGLM algorithm and $\left\{e_{1}, e_{2}\right\}$ by the dialytic step, with a global computation time of $17+227+670+567+340 \approx 1820 \mathrm{~min}$. Though this time seems very high, it represents a substantial achievement if the size of the resulting polynomial is taken into account. The authors are aware of no studies in which a resultant so large could be calculated on a normal PC. This proves the effectiveness of the proposed procedure.

The 216 roots of $G\left[I_{5}\right]$ may be complex or real. By varying the robot parameters, the count of real roots varies. Since there may be roots in the solution set that always remain complex, the maximal number of real solutions may be smaller than 216 . Determining a tight bound for this count is a challenging task. By a continuation procedure originally proposed by Dietmaier [21] and recently adapted by the authors to the DGP of underconstrained CDPRs [14], sets of parameters for which the DGP of the CDPR with 4 cables provides at the most 98 real configurations were found so far, e.g. $\mathbf{a}_{1}=[0 ; 0 ; 0], \mathbf{a}_{2}=[-0.76054 ; 0 ; 0.90931]$, $\mathbf{a}_{3}=[-0.71646 ; 0.68047 ; 0.07970], \mathbf{a}_{4}=[0.02574 ; 0.70420 ; 0.87389]$, $\mathbf{b}_{1}=[16.54820 ; 0 ; 0], \quad \mathbf{b}_{2}=[17.16360 ; 0.77720 ; 0], \quad \mathbf{b}_{3}=$ $[16.22250 ; 0.75153 ; 0.76874], \quad \mathbf{b}_{4}=[17.38600 ; 0.09567 ; 0.81726]$ and $\left(\rho_{1}, \rho_{2}, \rho_{3}, \rho_{4}\right)=(1 ; 1.01493 ; 1.12171 ; 1.138230)$. As it may be verified by the software described in Section V-C, DGP-Solver, in this case 20 configurations out of 98 have positive tension in all cables, and among these 5 are stable.

\section{DGP of CDPRs with 5 cables}

When 5 cables are taut, Eq. (1) provides 5 polynomials, i.e. $q_{i}$, $i=1 \ldots 5 . \mathbf{M}(P, \mathbf{X})$ is a $6 \times 6$ matrix, and only one equation may be obtained by imposing $\operatorname{rank}[\mathbf{M}(P, \mathbf{X})] \leq 6$. In this case, no additional linearly-independent equations emerge by changing the moment pole $P$, so that Eq. (10) reduces to

$$
p_{1}:=\operatorname{det} \mathbf{M}\left(A_{1}, \mathbf{X}\right)=0
$$

$I$ comprises 6 polynomials, i.e. $q_{1}, q_{2}, q_{3}, q_{4}, q_{5}$ and $p_{1}$, of degree 4 , $3,3,3,3$ and 9 in $\mathbf{X}$, respectively. The total number of monomials in $I$ is (as for the 4-cable CDPR) 1576. Though the computation of $G[I]$ 
TABLE I

Structure of THE GRoebNer BASES OF THE ELIMINATION IDEALS $\left\langle I_{l}\right\rangle$ OF $\langle I\rangle$ FOR A CDPR With 4 CABLES AND A GENERIC GEOMETRY

\begin{tabular}{ccccc}
\hline \hline$G\left[I_{l}\right]$ & $\mathbf{X} \backslash \mathbf{X}_{l}$ & $N_{l}$ & $\begin{array}{c}\text { Degrees of the generators } \\
\text { in } \mathbf{X} \backslash \mathbf{X}_{l}\end{array}$ & $\begin{array}{c}\text { No. of monomials with variables in } \\
\mathbf{X} \backslash \mathbf{X}_{l}-\{w\}, w \in \mathbf{X} \backslash \mathbf{X}_{l}\end{array}$ \\
\hline$G[I]$ & {$\left[z, y, x, e_{1}, e_{2}, e_{3}\right]$} & 195 & $3(3), 4(5), 5(158), 6(29)$ & $230,232,232,271,224,195$ \\
$G\left[I_{1}\right]$ & {$\left[y, x, e_{1}, e_{2}, e_{3}\right]$} & 147 & $5(36), 6(111)$ & $185,180,181,160,147$ \\
$G\left[I_{2}\right]$ & {$\left[x, e_{1}, e_{2}, e_{3}\right]$} & 111 & $5(1), 7(99), 8(11)$ & $127,127,117,111$ \\
$G\left[I_{3}\right]$ & {$\left[e_{1}, e_{2}, e_{3}\right]$} & 61 & $8(1), 10(60)$ & $66,61,61$ \\
$G\left[I_{4}\right]$ & {$\left[e_{2}, e_{3}\right]$} & 21 & $20(15), 21(6)$ & 22,21 \\
$G\left[I_{5}\right]$ & {$\left[e_{3}\right]$} & 1 & $216(1)$ & - \\
\hline
\end{tabular}

TABLE III

STRUCTURE OF THE GROEBNER BASES OF THE ELIMINATION IDEALS $\left\langle I_{l}\right\rangle$ OF $\langle I\rangle$ FOR A CDPR WITH 5 CABLES AND A GENERIC GEOMETRY

\begin{tabular}{ccccc}
\hline \hline \multirow{G}{*}[I_{l}]{} & $N_{l}$ & Degrees of the generators & No. of monomials with variables in \\
& $\mathbf{X} \backslash \mathbf{X}_{l}$ & & in $\mathbf{X} \backslash \mathbf{X}_{l}$ & $159,156,147,168,141,118$ \\
\hline$G[I]$ & {$\left[z, y, x, e_{1}, e_{2}, e_{3}\right]$} & 110 & $3(4), 4(46), 5(60)$ & $130,128,139,124,110$ \\
$G\left[I_{1}\right]$ & {$\left[y, x, e_{1}, e_{2}, e_{3}\right]$} & 110 & $4(6), 5(84), 6(20)$ & $87,96,83,69$ \\
$G\left[I_{2}\right]$ & {$\left[x, e_{1}, e_{2}, e_{3}\right]$} & 68 & $4(1), 5(4), 6(40), 7(23)$ & $62,43,31$ \\
$G\left[I_{3}\right]$ & {$\left[e_{1}, e_{2}, e_{3}\right]$} & 31 & $4(1), 8(11), 9(6), 10(6), 11(6), 12(1)$ & 18,17 \\
$G\left[I_{4}\right]$ & {$\left[e_{2}, e_{3}\right]$} & 17 & $16(13), 17(4)$ & - \\
$G\left[I_{5}\right]$ & {$\left[e_{3}\right]$} & 1 & $140(1)$ & \\
\hline \hline
\end{tabular}

TABLE II

COMPUTATION TIMES TO OBTAIN GROEBNER BASES OF THE ELIMINATION IDEALS $\left\langle I_{l}\right\rangle$ FOR A ROBOT WITH 4 CABLES AND $\mathbf{a}_{1}=[0 ; 0 ; 0], \mathbf{a}_{2}=[9 ; 0 ; 1], \mathbf{a}_{3}=[11 ; 9 ; 0], \mathbf{a}_{4}=[-2 ; 8 ;-1], \mathbf{b}_{1}=[-2 ;-1 ;-1]$, $\mathbf{b}_{2}=[1 ;-2 ; 0], \mathbf{b}_{3}=[2 ; 1 ;-1], \mathbf{b}_{4}=[0 ; 2 ;-1],\left(\rho_{1}, \rho_{2}, \rho_{3}, \rho_{4}\right)=(6,7,8,9)$.

\begin{tabular}{cccc}
\hline \hline$l$ & $\left\langle I_{l}\right\rangle$ & $T_{G\left[I_{l}\right]}[\mathrm{min}]$ & $T_{G\left[I_{5}\right]}[\mathrm{min}]$ \\
\hline 0 & $\langle I\rangle$ & 17 & $\ldots$ \\
1 & $\langle I\rangle \cap \mathbb{Q}\left[y, x, e_{1}, e_{2}, e_{3}\right]$ & 227 & $\ldots$ \\
2 & $\langle I\rangle \cap \mathbb{Q}\left[x, e_{1}, e_{2}, e_{3}\right]$ & 670 & $\ldots$ \\
3 & $\langle I\rangle \cap \mathbb{Q}\left[e_{1}, e_{2}, e_{3}\right]$ & 567 & 340 \\
4 & $\langle I\rangle \cap \mathbb{Q}\left[e_{2}, e_{3}\right]$ & 1063 & 67 \\
5 & $\langle I\rangle \cap \mathbb{Q}\left[e_{3}\right]$ & $\ldots$ & - \\
\hline \hline
\end{tabular}

may not take advantage of redundant generators in $I$, it is relatively fast, mainly because a single high-degree polynomial appears in $I$. For the exemplifying robot reported in subsequent Table IV, $G[I]$ ma be computed in roughly $3 \mathrm{~min}$.

The normal set, namely

$$
\mathbf{N}[I]=\left[1, e_{3}, e_{2}, e_{1}, x, y, z, e_{3}^{2}, e_{2} e_{3}, \ldots, e_{1} x y^{2}, e_{1} x y z, x^{4}\right]^{T}
$$

contains 140 monomials, so that $N_{\text {sol }}=140$.

The structure of $G\left[I_{l}\right]$ with respect to grevlex $\left(\mathbf{X} \backslash \mathbf{X}_{l}\right)$ is reported in Table III for $l=0 \ldots 5$. The table is constructed as Table I. Notably, in this case, $G\left[I_{l}\right]$ comprises a number of monomials in $\mathbf{X} \backslash \mathbf{X}_{l}-\left\{e_{3}\right\}$ equal to $N_{l}$ only for $l=1,3,4$. Hence, Sylvester dialytic elimination may be applied to neither $G[I]$ nor $G\left[I_{2}\right]$. Indeed, these contain more monomials in $\mathbf{X} \backslash \mathbf{X}_{l}-\left\{e_{3}\right\}$ than available generators.

Table IV reports, for an exemplifying generic robot, the CPU time $T_{G\left[I_{l}\right]}$ required to compute $G\left[I_{l}\right]$ from $\left\langle I_{l-1}\right\rangle$, and the CPU time $T_{G\left[I_{5}\right]}$ to calculate $G\left[I_{5}\right]$ by applying Sylvester's elimination to $G\left[I_{l}\right]$, for $l=1,3,4$. The most efficient computation is obtained again by eliminating $\{x, y, z\}$ via the FGLM algorithm and $\left\{e_{1}, e_{2}\right\}$ via a dialytic step $(3.3+43.0+59.5+56.7+7.5 \approx 170 \mathrm{~min}) . G\left[I_{3}\right]$ comprises 31 polynomials ( 1 of degree 4,11 of degree 8,6 of degree $9,6$ of degree 10,6 of degree 11 and 1 of degree 12 in $\Phi)$, and these include 31 monomials in $e_{1}$ and $e_{2}$. Hence, a 140-degree polynomial in $e_{3}$ may be computed by expanding a $31 \times 31$ eliminant matrix.

By the continuation procedure presented in [14], several sets of geometric parameters for which the DGP provides at the most 74 real configurations have been found so far. An example is as follows: $\mathbf{a}_{2}=[1.44417,0,1.20333], \mathbf{a}_{3}=[0.302415,1.26206,0.55533]$, $\mathbf{a}_{4}=[-0.711127,0.808726,0.810451], \mathbf{a}_{5}=[0.749568,0.761578,-$ $0.469085], \mathbf{b}_{1}=[2.16169,0,0], \mathbf{b}_{2}=[-0.125711,0,1.32615], \mathbf{b}_{3}=$ $[-0.412791,0.0211425,0.449869], \mathbf{b}_{4}=[-0.16265,-0.468249,-$ $0.399945], \quad \mathbf{b}_{5}=[1.59653,1.31446,0.96224],\left(\rho_{1}, \rho_{2}, \rho_{3}, \rho_{4}, \rho_{5}\right)=$ $(2.46449,1.99586,1.20622,1.42395,2.4302)$. As it may be verified by DGP - Solver, 3 configurations out of 74 have positive tension in all cables, and among these 2 are stable.

\section{TABLE IV}

COMPUTATION TIMES TO OBTAIN GROEBNER BASES OF THE ELIMINATION IDEALS $\left\langle I_{l}\right\rangle$ FOR A ROBOT WITH 5 CABLES AND

$$
\begin{gathered}
\mathbf{a}_{1}=[0 ; 0 ; 0], \mathbf{a}_{2}=[1 ; 2 ;-0.75], \mathbf{a}_{3}=[3.5 ; 1 ; 1], \mathbf{a}_{4}=[3.25 ;-1 ; 1], \mathbf{a}_{5}= \\
{[1 ;-2 ;-0.5], \mathbf{b}_{1}=[-1 ; 0 ;-1], \mathbf{b}_{2}=[-0.5 ; 1 ;-1.25], \mathbf{b}_{3}=} \\
{[0.75 ; 0.75 ;-1.25], \mathbf{b}_{4}=[0.5 ;-0.75 ;-1.25], \mathbf{b}_{5}=} \\
{[-0.25 ;-0.8 ;-1.5],\left(\rho_{1}, \rho_{2}, \rho_{3}, \rho_{4}, \rho_{5}\right)=(4.5 ; 5 ; 3 ; 3.75 ; 4.75) .}
\end{gathered}
$$

\begin{tabular}{cccc}
\hline \hline$l$ & $\left\langle I_{l}\right\rangle$ & $T_{G\left[I_{I}\right]}[\mathrm{min}]$ & $T_{G\left[I_{5}\right]}[\mathrm{min}]$ \\
\hline 0 & $\langle I\rangle$ & 3.3 & $\ldots$ \\
1 & $\langle I\rangle \cap \mathbb{Q}\left[y, x, e_{1}, e_{2}, e_{3}\right]$ & 43.0 & 4042 \\
2 & $\langle I\rangle \cap \mathbb{Q}\left[x, e_{1}, e_{2}, e_{3}\right]$ & 59.5 & $\ldots$ \\
3 & $\langle I\rangle \cap \mathbb{Q}\left[e_{1}, e_{2}, e_{3}\right]$ & 56.7 & 7.5 \\
4 & $\langle I\rangle \cap \mathbb{Q}\left[e_{2}, e_{3}\right]$ & 73.0 & 10.7 \\
5 & $\langle I\rangle \cap \mathbb{Q}\left[e_{3}\right]$ & $\ldots$ & - \\
\hline \hline
\end{tabular}




\section{DGP of CDPRs with 6 cables}

When 6 cables are taut, Eq. (1) provides 6 equations and the geometric constraints are sufficient to completely determine the platform pose. Kinematics and statics are decoupled and the DGP is equivalent to the forward displacement analysis of the generalized Gough-Stewart manipulator, with the latter being governed by 6 relations identical to those in Eq. (1). This problem admits 40 solutions in the complex field, all of which may be real [21], and algorithms for their computation are well known [25].

\section{NUMERICAL COMPUTATION OF THE SOLUTION SET}

For the numeric calculation of the solution set, working with polynomials of degree as high as 156,216 or 140 is unpractical. On the other hand, once a Groebner basis $G[I]$ of $\langle I\rangle$ is known, the solutions of the DGP may be efficiently computed from the eigenpairs of a $N_{\text {sol }} \times N_{\text {sol }}$ numeric matrix $\mathbf{A}\left[I, e_{3}\right]$, called multiplication matrix [27], which may be obtained from the the normal set $\mathbf{N}[I]$. Details are in [12]. A drawback of this approach is that it relies on a prior computation of $G[I]$. The efficiency of the computation of a Groebner basis highly depends on the 'size' of the rational coefficients of the polynomials generating the basis. Indeed, when the coefficients are obtained by converting real values, the higher the number of digits in the original floating-point data, the bigger the numerators and denominators of the resulting rationals. As a result, computation becomes slower and memory-demanding. This is a limitation shared by all computational methods based on Groebner bases. This is the reason why homotopy continuation [25] is chosen in this paper to actually numerically compute the solution set. Continuation has the significant advantage that it requires no prior Groebner-basis computation by a computer algebra system and real-value geometric parameters may be directly handled in floating-point arithmetic. As a consequence, the dependence of computation time on the specific values of the robot parameters is rather modest.

While formulating equilibrium constraints via Eq. (10) is particularly favorable when elimination strategies are pursued, for continuation algorithms the complexity and the high degree of the polynomial emerging from the elimination of cable tensions are a disadvantage, since they slow down computation and cause stability problems. For this reason, the formulation of the DGP via Eq. (1) and (2) is preferable. For the same reason, the pose parametrization by Study or Dietmaier coordinates (see Sections II-C and II-D) is preferable over the 6-parameter representation described in Section II-B. Though the resulting system involves more variables and more equations than the one used in Section III, it comprises much simpler lowerorder polynomials, which are stabler when homotopy continuation is implemented, thus leading to a faster computation.

\section{A. Homotopy-continuation algorithms}

Polynomial homotopy continuation is a path-tracking technique that transforms a start system of polynomial equations with known solutions to the target system whose solutions has to be found [25]. Depending on how the start system is constructed, the procedure may be classified as general homotopy continuation or parameter homotopy continuation. The former is employed when no information is a priori known about the roots of the target system. In this case, Study coordinates are chosen to parametrize the platform pose, since they lead to the lowest multi-homogeneous Bezout number $N_{B e z}$, which coincides with the number of paths to be tracked. However, since $N_{B e z}$ is still much larger than $N_{\text {sol }}$ (for the robots with 2, 3, 4, 5 and 6 cables, $N_{B e z}$ is equal to, respectively, 96, 5120, 3840, 1536 and 256), many paths diverge to infinity.
When the isolated roots of the DGP are known for a generic robot geometry, parameter homotopy continuation may be used to find solutions for any other target robot of the same family. In this case, the burden of tracking paths diverging to infinity is avoided. When implementing parameter continuation, Dietmaier parametrization is preferred over Study one. In the latter case, in fact, the number of isolated roots of the equations governing the DGP is $2 N_{\text {sol }}$ (since Study coordinates $\mathbf{S}$ and $-\mathbf{S}$ represent the same platform pose, see Eqs. (5)-(6)) and so this is also the number of paths to be tracked for parameter homotopy to work robustly. This problem does not appear if Dietmaier parametrization is used, as in this case the number of isolated roots of the modeling equations is exactly equal to $N_{\text {sol }}$.

\section{B. Equilibrium configurations with slack cables}

When cable lengths are assigned as inputs, nothing ensures, a priori, that when the platform reaches its stable equilibrium pose all cables are in tension, since configurations may exist in which the platform is supported by only $m$ cables, with $m \leq n$ and with $n-m$ cables being slack. Accordingly, the overall solution set emerges by solving the DGP for all possible constraint sets $\left\{\left\|\mathbf{s}_{j}\right\|=\rho_{j}, j \in \mathcal{W}\right\}$, with $\mathcal{W} \subseteq\{1 \ldots n\}$ and $\operatorname{card}(\mathcal{W}) \leq n$. As an example, for a robot with 5 cables, 31 DGPs need to be solved, namely 1 DGP with 5 active cables, 5 DGPs with 4 active cables, 10 DGPs with 3 active cables, 10 DGPs with 2 active cables, and 5 DGPs with 1 active cable. Clearly, when the $k$ th cable is slack, the distance $\left\|\mathbf{s}_{k}\right\|$ cannot be greater than the assigned $\rho_{k}$. Hence, for any subset $\mathcal{W}$, only the solutions for which $\left\|\mathbf{s}_{k}\right\| \leq \rho_{k}$, for all $k \notin \mathcal{W}$, are admissible and must be retained.

\section{DGP-Solver}

DGP - Solver is a software based on the procedures outlined in Sections V-A and V-B. It is available for both Linux and Windows platforms, and it is freely distributed [16]. DGP - Solver solves the DGP for a generic $n$-cable CDPR, with $n \leq 6$. It receives the robot geometry, the cable lengths and a constant external load as inputs, and it computes all possible equilibrium configurations that are compatible with the given constraints $\left(\left\|\mathbf{s}_{i}\right\| \leq \rho_{i}, \tau_{i} \geq 0, i=1 \ldots n\right)$, including those with slack cables. Upper limits on cable tensions are not taken into consideration in the current version of the software. DGP - Solver also determines whether an equilibrium configuration is stable or not, by assessing the definiteness of the corresponding reduced Hessian $\mathbf{H}_{r}$ [11].

In recent years, several softwares have been developed to ease the implementation of homotopy-continuation algorithms. DGP - Solver uses Bertini [17] as a computation engine. The advantage of Bertini over other packages relies on its capability of implementing user-defined parameter homotopies. DGP - Solver uses both parameter and general continuation routines. The former provides for the fastest computation, but some (normally complex) solutions may sometimes be missed. When this happen, DGP - Solver uses general continuation to correct the outcome.

Tables V and Table VI show the results provided by DGP - Solver for two exemplifying CDPRs with, respectively, 4 and 5 cables (extracted from the samples discussed in subsequent Section VI). Due to space limitations, only the real solutions with nonnegative tension in all cables are reported (the platform orientation is specified by Rodrigues parameters, whereas symbols $>$ and $\langle>$ denote, respectively, a positive-definite and an indefinite Hessian matrix). The 4-cable robot in Table $\mathrm{V}$ has 4 poses with only two cables in tension, all of them unstable, and 2 stable configurations, i.e. 1 with three active cables and 1 with all cables taut. The 5-cable robot in 


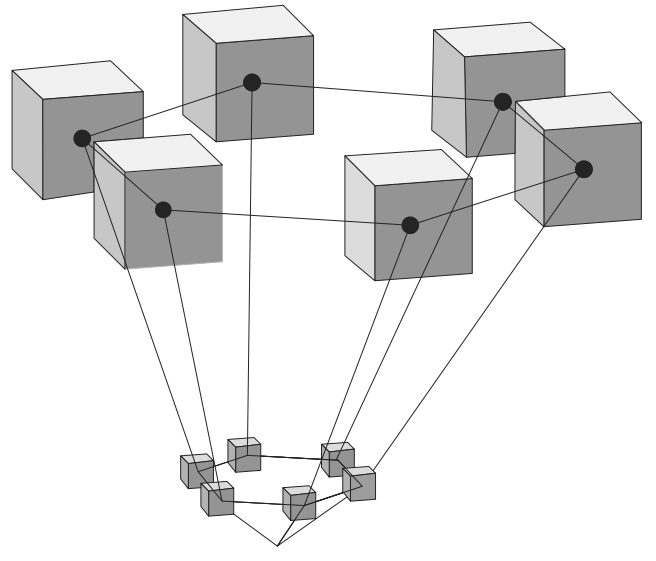

Fig. 2. Schematic of the robot samples used for the stochastic investigation.

Table VI has 9 unstable poses with two, three or four taut cables, and 4 stable configurations, 2 with four and 2 with five cables in tension.

When the DGP admits multiple feasible solutions, the robot may switch (because of inertia forces or external disturbances) across portions of the configuration space characterized by different numbers of taut cables, thus bringing the end-effector onto unpredicted trajectories. Accordingly, the computation of the complete set of equilibrium configurations is essential for robust trajectory planning.

\section{APPLICATION: Design OF CDPRs}

A robot with $n$ cables is designed to control $n$ dofs of the platform. However, depending on the configuration and the load, only $m$ cables may be active, with $m<n$. Slack cables contribute to neither controlling the platform pose nor sustaining the load, thus being instantaneously ineffectual. This amounts to a loss of robot capacity. Investigating the most suitable architectures and geometries that guarantee the optimal exploitation of the available actuators is in order. A stochastic investigation is reported hereafter providing preliminary ground in this respect.

Two scenarios are envisaged. In one case, the robot geometry is established, and the platform configuration and the load orientation are changed. Alternatively, the load is assigned, and the robot geometry and configuration are varied. Since the two scenarios are equivalent, the latter is described in the following. For the sake of simplicity, the anchor points on the base and the platform are chosen inside cubes having center-points located on the vertexes of regular hexagons (Fig. 2). The circumradii of the base and platform hexagons are 10 and 3.5, respectively, whereas the side lengths of the base and platform cubes are 4 and 1, respectively. $G$ is located on the line that is perpendicular to the platform hexagon and that passes through its center, at a distance of 1.5 from the latter. Cable lengths are varied in the interval $[10,16] .500$ robot configurations are randomly chosen within the above limits, thus generating 500 samples of 6cable robots. For each sample, DGP - Solver computes the overall solution set $\mathcal{S}$. The latter comprises all solutions of the DGP such that $\left\{\left\|\mathbf{s}_{i}\right\| \leq \rho_{i}, i=1 \ldots 6\right\}$, where the equality holds for taut cables and the inequality for slack ones. By ignoring the constraint $\left\|\mathbf{s}_{i}\right\| \leq \rho_{i}$ for $6-k$ cables, $500\left(\begin{array}{l}6 \\ k\end{array}\right)$ additional samples of $k$-cable robots, with $k=2 \ldots 5$, may be easily extracted from DGP - Solver computation ${ }^{4}$.

\footnotetext{
${ }^{4}$ As explained in Section V-B, DGP - Solver provides the DGP solutions for all possible constraint sets $\mathcal{C}_{\mathcal{W}}=\left\{\left\|\mathbf{s}_{j}\right\|=\rho_{j}, \tau_{j}>0, j \in \mathcal{W}\right\}$, with $\mathcal{W} \subseteq$ $\{1,2,3,4,5,6\}, m=\operatorname{card}(\mathcal{W}) \leq 6$, and $\left\{\left\|\mathbf{s}_{k}\right\| \leq \rho_{k}, \forall k \notin \mathcal{W}\right\}$. By ignoring the latter requirement, the DGP solutions for $\mathcal{C}_{\mathcal{W}}$ provide the equilibrium configurations of a robot including only $m$ cables.
}

The results of the simulation are summarized in Table VII. The $n$th column, with $n=2 \ldots 6$, reports the results obtained for the samples suspended by $n$ cables, and in particular: the number of samples considered (row 1), the number of feasible configurations globally obtained (row 2), and the number of feasible configurations with $m$ cables in tensions, $m \leq n$ (row $2+m$ ).

The data emerging from the table seem to show that, when the number of cables increases, the probability of finding feasible configurations with all cables in tension decreases. For the robots with 2 cables, in almost $90 \%$ of equilibrium configurations both cables are active, whereas for the robots with 3 cables all cables are active in roughly $80 \%$ of cases. This trend continues as $n$ increases, until, for the samples with 6 cables, in almost no configuration all 6 cables are active. Columns 4 through 6 show that, in most cases, the robots with 4 cables have only 3 taut cables, whereas the robots with 5 and 6 cables have only 3 or 4 cables in tension. It is worth observing that, while for the robots with 2 or 3 cables equilibrium configurations with slack cables may only occur at the frontier of the geometrical workspace $^{5}$, it is not so when $n \geq 4$, where equilibrium poses with slack cables may occur in the middle of it.

Another interesting question concerns the probability for a CDPR to admit multiple feasible configurations. When this occurs, the stable equilibrium pose of the platform may change under the influence of external perturbations, which is not favorable in practice. In this perspective, Table VIII investigates how many multiple solutions are encountered when solving the DGP of the samples in Table VII. Row 2, 3 and 4 report, respectively, the percentages of samples admitting 1,2 or more feasible solutions. It emerges that when the number of cables increases, the probability of having a single solution of the DGP decreases. It is roughly $83 \%$ for the 3 -cable robots, $53 \%$ for the 4-cable samples, and a little less than $25 \%$ for the 5 - and the 6-cable robots.

From the data reported in Tables VII and VIII, one could argue that, as long as only 3 cables are employed, all of them may be reasonably expected to support the platform, with a single stable equilibrium pose being unambiguously determined in most circumstances. When 4, 5 or 6 cables are used, a full control of the robot becomes challenging, because it is difficult to take advantage of all available cables and to control the platform pose in a deterministic way.

\section{CONCLUSIONS}

This paper studied the direct geometrico-static problem (DGP) of underconstrained cable-driven parallel robots (CDPRs) supported by $n$ cables, with $n \leq 6$. The task consisted in finding the equilibrium configurations of the end-effector when cable lengths and a static load are assigned.

The problem was modeled by a set of algebraic equations, and a least-degree univariate polynomial in the corresponding ideal was found for any value of $n$, thus setting an exact bound on the number of solutions admitted in the complex field. The most challenging tasks had to be solved for $n=3,4$ and 5, when the aforementioned polynomial has degree 156, 216 and 140, respectively. The proposed variable-elimination procedure succeeded when other methods proved to be computationally too onerous, thus providing an efficient alternative to the state of the art in calculating a least-degree univariate polynomial in a given ideal.

For the numerical computation of the solution set, a numerical procedure based on homotopy continuation was developed and implemented in the software DGP - Solver. Distinctive features of the code

\footnotetext{
${ }^{5}$ When a single cable is taut, the external wrench $Q \mathcal{L}_{e}$ must be aligned with it. When $n=3$ and only two cables are taut, the latter and $Q \mathcal{L}_{e}$ must be coplanar $[11,12]$.
} 
TABLE V

DGP OF A CDPR WITH 4 CABLES: REAL SOLUTIONS WITH NONNEGATIVE TENSION IN ALL CABLES

Geometric dimensions and load: $\mathbf{a}_{1}=[5.521454,7.836054,-1.009788], \mathbf{a}_{2}=[-5.366081,8.252356,1.959491], \mathbf{a}_{3}=[-10.315057,1.612391,0.946641]$, $\mathbf{a}_{4}=[5.392802,-7.491653,0.579554], \mathbf{b}_{1}=[2.182515,3.334434,1.997996], \mathbf{b}_{2}=[-2.214432,3.330068,1.013029], \mathbf{b}_{3}=[-3.659557,0.265737,1.111276]$, $\mathbf{b}_{4}=[1.404915,-3.195786,1.219710],\left(\rho_{1}, \rho_{2}, \rho_{3}, \rho_{4}\right)=(14.549082,14.549329,15.763856,10.898894), Q=10$.

\begin{tabular}{|c|c|c|c|c|}
\hline Conf. & $(x, y, z)$ & $\left(e_{0}, e_{1}, e_{2}, e_{3}\right)$ & $\left(\tau_{1}, \tau_{2}, \tau_{3}, \tau_{4}\right)$ & $\mathbf{H}_{r}$ \\
\hline 1 & $(5.4865,3.6679,8.6012)$ & $(1,1.1961,-0.4459,-0.5447)$ & $(9.16,0,0,5.32)$ & $<>$ \\
\hline 2 & $(5.4514,-0.5145,9.2931)$ & $(1,0.0413,-0.4465,-0.0290)$ & $(5.09,0,0,5.57)$ & $<>$ \\
\hline 3 & $(5.4947,4.6478,8.5797)$ & $(1,0.7274,-0.4462,-0.3354)$ & $(8.77,0,0,3.49)$ & $<>$ \\
\hline 4 & $(-3.0150,-2.6186,10.5744)$ & $(1,-0.2066,0.7251,0.3046)$ & $(0,0,6.19,5.71)$ & $<>$ \\
\hline 5 & $(-0.4245,-1.7527,11.0969)$ & $(1,-1.4031,1.8469,0.2283)$ & $(3.34,0,4.63,5.20)$ & $>$ \\
\hline 6 & $(-0.1964,-0.1268,11.0728)$ & $(1,0.2101,0.3801,0.0573)$ & $(2.89,0.30,3.92,4.48)$ & $>$ \\
\hline
\end{tabular}

TABLE VI

DGP OF A CDPR WITH 5 CABLES: REAL SOLUTIONS WITH NONNEGATIVE TENSION IN ALL CABLES

Geometric dimensions and load: $\mathbf{a}_{1}=[8.762837,-1.064001,-0.715711], \mathbf{a}_{2}=[6.732934,8.223691,0.187221], \mathbf{a}_{3}=[-5.094292,7.798299,1.258330], \mathbf{a}_{4}=$ $[-11.189309,-0.138832,-1.614663], \mathbf{a}_{5}=[4.870417,-8.101810,0.176616], \mathbf{b}_{1}=[3.889210,0.116354,1.549903], \mathbf{b}_{2}=[1.262183,3.258968,1.975178]$, $\mathbf{b}_{3}=[-1.533217,2.692867,1.915029], \mathbf{b}_{4}=[-3.991803,0.020197,1.186929], \mathbf{b}_{5}=[1.257342,-3.138215,1.992397],\left(\rho_{1}, \rho_{2}, \rho_{3}, \rho_{4}, \rho_{5}\right)=(14.772834$, $12.755196,13.153812,13.969011,15.712028), Q=10$.

\begin{tabular}{|c|c|c|c|c|}
\hline Conf. & $(x, y, z)$ & $\left(e_{0}, e_{1}, e_{2}, e_{3}\right)$ & $\left(\tau_{1}, \tau_{2}, \tau_{3}, \tau_{4}, \tau_{5}\right)$ & $\mathbf{H}_{r}$ \\
\hline 1 & $(-1.8836,-0.5703,3.6644)$ & $(1,1.9122,103.4291,-2.6272)$ & $(20.26,0,0,20.86,0)$ & $<>$ \\
\hline 2 & $(-0.9683,4.6303,8.1748)$ & $(1,0.4483,0.1963,0.0244)$ & $(0,6.19,0,5.44,0)$ & $<>$ \\
\hline 3 & $(-0.5205,0.5002,9.6903)$ & $(1,-22.1474,-12.1484,-1.3686)$ & $(0,0,10.24,0,10.34)$ & $<>$ \\
\hline 4 & $(-2.2633,3.8189,4.8682)$ & $(1,14.9241,-32.9020,16.6404)$ & $(0.47,21.60,0,22.88,0)$ & $<>$ \\
\hline 5 & $(-2.1884,2.2735,4.9241)$ & $(1,4131.8466,-12513.9896,3408.9760)$ & $(4.72,13.77,0,18.94,0)$ & $<>$ \\
\hline 6 & $(1.5688,-3.0697,10.5834)$ & $(1,-0.5711,-0.3564,-0.1105)$ & $(1.10,1.85,0,3.66,5.40)$ & $<>$ \\
\hline 7 & $(1.3853,-2.5305,10.5848)$ & $(1,-0.4701,-0.2959,-0.0865)$ & $(1.22,2.02,0,3.72,4.87)$ & $>$ \\
\hline 8 & $(-1.7403,0.4235,10.0329)$ & $(1,24.6125,21.5424,3.9457)$ & $(1.44,0,6.64,2.85,7.07)$ & $<>$ \\
\hline 9 & $(-2.5658,2.0842,10.1100)$ & $(1,2.8702,2.2467,0.5195)$ & $(0,1.62,5.36,3.23,6.15)$ & $<>$ \\
\hline 10 & $(1.5754,-2.4698,10.6232)$ & $(1,-1.6897,-0.3362,-0.1492)$ & $(0,3.75,0.50,3.17,6.71)$ & $>$ \\
\hline 11 & $(1.5476,-3.5523,10.5849)$ & $(1,-1.0357,-0.4345,-0.1603)$ & $(0,2.02,1.14,2.90,7.12)$ & $<>$ \\
\hline 12 & $(-2.6029,1.9238,10.1101)$ & $(1,3.0172,2.4254,0.5732)$ & $(0.02,1.54,5.32,3.34,6.24)$ & $>$ \\
\hline 13 & $(1.5460,-3.4460,10.6187)$ & $(1,-0.9363,-0.6196,-0.1883)$ & $(0.60,1.70,0.77,3.52,6.53)$ & $>$ \\
\hline
\end{tabular}

TABLE VII

STOCHASTIC ANALYSIS OF RANDOM SAMPLES OF ROBOT GEOMETRIES WITH 2, 3, 4, 5 AND 6 CABLES.

\begin{tabular}{|c|c|c|c|c|c|}
\hline$n$-cable CDPRs & 2-cable CDPRs & 3-cable CDPRs & 4-cable CDPRs & 5-cable CDPRs & 6-cable CDPRs \\
\hline No. of samples & 7500 & 10000 & 7500 & 3000 & 500 \\
\hline No. of feasible solutions & 7500 & 12048 & 12006 & 5912 & 1114 \\
\hline No. of feasible solutions with 1 active cable & $785(10.46 \%)$ & $70(0.58 \%)$ & 0 & 0 & 0 \\
\hline No. of feasible solutions with 2 active cables & $6715(89.53 \%)$ & $2424(20.11 \%)$ & $109(0.90 \%)$ & $3(0.05 \%)$ & 0 \\
\hline No. of feasible solutions with 3 active cables & - & $9554(79.29 \%)$ & $6987(58.19 \%)$ & $2362(39.95 \%)$ & $380(34.11 \%)$ \\
\hline No. of feasible solutions with 4 active cables & - & - & $4910(40.89 \%)$ & $2626(44.41 \%)$ & $420(37.70 \%)$ \\
\hline No. of feasible solutions with 5 active cables & - & - & - & $921(15.57 \%)$ & $312(28.00 \%)$ \\
\hline No. of feasible solutions with 6 active cables & - & - & - & - & $2(0.17 \%)$ \\
\hline
\end{tabular}

TABLE VIII

DISTRIBUTION OF MULTIPLE FEASIBLE CONFIGURATIONS AS EMERGING FROM THE STOCHASTIC ANALYSIS.

\begin{tabular}{cccccc}
\hline \hline$n$-cable CDPRs & 2-cable CDPRs & 3-cable CDPRs & 4-cable CDPRs & 5-cable CDPRs & 6-cable CDPRs \\
\hline No. of samples & 7500 & 10000 & 7500 & 4000 & 500 \\
No. of samples with 1 feasible solution & $7500(100 \%)$ & $8320(83.20 \%)$ & $3941(52.54 \%)$ & $988(24.70 \%)$ & $113(22.60 \%)$ \\
No. of samples with 2 feasible solutions & 0 & $1299(12.99 \%)$ & $2754(36.72 \%)$ & $1276(31.90 \%)$ & $213(42.60 \%)$ \\
No. of samples with more than 2 feasible solutions & 0 & $381(3.81 \%)$ & $805(10.73 \%)$ & $736(18.40 \%)$ & $174(34.80 \%)$ \\
\hline \hline
\end{tabular}


are that all solutions of the problem are found, including those with slack cables, and stability analysis is integrated to identify feasible equilibria.

It was shown that the DGP may admit multiple equilibrium configurations, characterized by a different number of taut cables. Since slack cables are inactive, they represent a loss of capacity of the robot, which is instantaneously unable to control some of the end-effector freedoms. A preliminary investigation was performed to assess the most suitable architectures that may guarantee an optimal exploitation of the available actuators. The probability for a CDPR to admit multiple stable equilibrium configurations (which is a critical situation for the reliable control of the robot) was also considered. The latter issues deserve a deeper investigation, and they will be the topic of future research.

\section{ACKNOWLEDGMENT}

The authors express their gratitude to the Bertini team for granting permission to use their software. The financial support by the Italian Ministry of Education, Universities and Research through the PRIN grant No. 20124SMZ88_002 is gratefully acknowledged.

\section{REFERENCES}

[1] M. Yamamoto, N. Yanai, and A. Mohri, “Trajectory control of incompletely restrained parallel-wire-suspended mechanism based on inverse dynamics," IEEE Transactions on Robotics, vol. 20, no. 5, pp. 840-850, 2004.

[2] A. Fattah and S. K. Agrawal, "On the design of cable-suspended planar parallel robots," ASME Journal of Mechanical Design, vol. 127, no. 5, pp. 1021-1028, 2006.

[3] T. Heyden and C. Woernle, "Dynamics and flatness-based control of a kinematically undetermined cable suspension manipulator," Multibody System Dynamics, vol. 16, no. 2, pp. 155177, 2006.

[4] Q. Jiang and V. Kumar, "The direct kinematics of objects suspended from cables," in ASME 2010 Int. Design Engineering Technical Conferences, vol. 2, Montreal, Canada, August 15-18 2010, pp. 193-202, Paper no. DETC2010-28036.

[5] B. Gao, J. Xu, J. Zhao, and N. Xi, "Combined inverse kinematic and static analysis and optimal design of a cable-driven mechanism with a spring spine," Advanced Robotics, vol. 26, no. 8-9, pp. 923-946, 2012.

[6] J.-F. Collard and P. Cardou, "Computing the lowest equilibrium pose of a cable-suspended rigid body," Optimization and Engineering, vol. 14, no. 3, pp. 457-476, 2013.

[7] J.-P. Merlet, "Wire-driven parallel robot: Open issues," in Romansy 19 - Robot Design, Dynamics and Control, V. Padois, P. Bidaud, and O. Khatib, Eds. Springer, 2013, pp. 3-10.

[8] S. Bouchard, C. Gosselin, and B. Moore, "On the ability of a cable-driven robot to generate a prescribed set of wrenches," ASME Journal of Mechanisms and Robotics, vol. 2, no. 1, pp. 011010/1-10, 2010.

[9] M. Gouttefarde, D. Daney, and J.-P. Merlet, "Interval-analysisbased determination of the wrench-feasible workspace of parallel cable-driven robots,' IEEE Transactions on Robotics, vol. 27, no. 1, pp. 1-13, 2011.

[10] M. Carricato and J.-P. Merlet, "Geometrico-static analysis of under-constrained cable-driven parallel robots," in Advances in Robot Kinematics: Motion in Man and Machine, J. Lenarčič and M. M. Stanišic̀, Eds. Springer, 2010, pp. 309-319.

[11] _ _ "Stability analysis of underconstrained cable-driven parallel robots," IEEE Transactions on Robotics, vol. 29, no. 1, pp. 288-296, 2013.
[12] M. Carricato, "Direct geometrico-static problem of underconstrained cable-driven parallel robots with three cables," ASME Journal of Mechanisms and Robotics, vol. 5, no. 3, pp. 031008/1-10, 2013.

[13] — , "Inverse geometrico-static problem of under-constrained cable-driven parallel robots with three cables," ASME Journal of Mechanisms and Robotics, vol. 5, no. 3, pp. 031002/1-11, 2013.

[14] G. Abbasnejad and M. Carricato, "Real solutions of the direct geometrico-static problem of under-constrained cable-driven parallel robots with 3 cables: a numerical investigation," Meccanica, vol. 47, no. 7, pp. 1761-1773, 2012.

[15] J.-P. Merlet, "Further analysis of the 2-2 wire-driven parallel crane," in Proc. of the 6th Int. Workshop on Computational Kinematics, F. Thomas and A. Perez Gracia, Eds. Barcelona, Spain, May 12-15: Springer, 2013, pp. 9-16.

[16] G. Abbasnejad and M. Carricato, "DGP-Solver: Software for the direct geometrico-static analysis of cable-driven parallel robots," http://grab.diem.unibo.it/DGP-Solver/.

[17] D. J. Bates, J. D. Hauenstein, A. J. Sommese, and C. W. Wampler, "Bertini: Software for numerical algebraic geometry," available at http://bertini.nd.edu.

[18] M. Carricato and G. Abbasnejad, "Direct geometrico-static analysis of under-constrained cable-driven parallel robots with 4 cables," in Proc. of the 1st Int. Conference on Cable-Driven Parallel Robots, T. Bruckmann and A. Pott, Eds. Stuttgart, Germany, Sept. 2-4: Springer, 2012, pp. 269-285.

[19] G. Abbasnejad and M. Carricato, "Direct geometrico-static problem of underconstrained cable-driven parallel robots with 5 cables," in Proc. of the 6th Int. Workshop on Computational Kinematics, F. Thomas and A. Perez Gracia, Eds. Barcelona, Spain, May 12-15: Springer, 2013, pp. 49-56.

[20] O. Bottema and B. Roth, Theoretical kinematics. Dover Publications, 1990.

[21] P. Dietmaier, "The Stewart-Gough platform of general geometry can have 40 real postures," in Advances in Robot Kinematics: Analysis and Control, J. Lenarčič and M. L. Husty, Eds. Kluwer Academic Publishers, 1998, pp. 7-16.

[22] D. Cox, J. Little, and D. O'Shea, Ideals, varieties, and algorithms. Springer, 2007.

[23] M. Raghavan and B. Roth, "Solving polynomial systems for the kinematic analysis and synthesis of mechanisms and robot manipulators," ASME Journal of Mechanical Design, vol. 117, no. 2B, pp. 71-79, 1995.

[24] J.-C. Faugère and Y. Liang, "Pivoting in extended rings for computing approximate Gröbner bases," Mathematics in Computer Science, vol. 5, no. 2, pp. 179-194, 2011.

[25] A. J. Sommese and C. W. Wampler, The numerical solution of systems of polynomials arising in engineering and science. World Scientific Publishing, 2005.

[26] A. K. Dhingra, A. N. Almadi, and D. Kohli, "A GröbnerSylvester hybrid method for closed-form displacement analysis of mechanisms," ASME Journal of Mechanical Design, vol. 122, no. 4, pp. 431-438, 2000.

[27] R. M. Corless, "Gröbner bases and matrix eigenproblems," $A C M$ SIGSAM Bulletin, vol. 30, no. 4, pp. 26-32, 1996.

[28] J. C. Faugère, P. Gianni, D. Lazard, and T. Mora, "Efficient computation of zero-dimensional Gröbner bases by change of ordering," Journal of Symbolic Computation, vol. 16, no. 4, pp. 329-344, 1993. 